Editorial

\title{
Computation: A New Open Access Journal of Computational Chemistry, Computational Biology and Computational Engineering
}

\author{
Karlheinz Schwarz $^{1, *}$, Rainer Breitling ${ }^{2}$ and Christian Allen ${ }^{3}$
}

1 Institute for Materials Chemistry, Vienna University of Technology, A-1060 Vienna, Getreidemarkt 9/165, Austria

2 Manchester Institute of Biotechnology, University of Manchester, 131 Princess Street, Manchester, M1 7DN, UK; E-Mail: rainer.breitling@manchester.ac.uk

3 Department of Aerospace Engineering, University of Bristol, BS8 1TR, UK;

E-Mail: c.b.allen@bristol.ac.uk

* Author to whom correspondence should be addressed; E-Mail: kschwarz@theochem.tuwien.ac.at; Tel.: +43-1-58801-165301; Fax: +43-1-58801-165982.

Received: 15 July 2013; in revised form: 22 August 2013 / Accepted: 22 August 2013 /

Published: 4 September 2013

\section{Introduction}

Computation (ISSN 2079-3197; http://www.mdpi.com/journal/computation) is an international scientific open access journal focusing on fundamental work in the field of computational science and engineering. Computational science has become essential in many research areas by contributing to solving complex problems in fundamental science all the way to engineering. The very broad range of application domains suggests structuring this journal into three sections, which are briefly characterized below. In each section a further focusing will be provided by occasionally organizing special issues on topics of high interests, collecting papers on fundamental work in the field. More applied papers should be submitted to their corresponding specialist journals. To help us achieve our goal with this journal, we have an excellent editorial board to advise us on the exciting current and future trends in computation from methodology to application. We very much look forward to hearing all about the research going on across the world. 


\section{Computational Chemistry}

The field of computational chemistry is becoming more and more important as the profession moves from basic questions in methodology to realistic simulations of complex materials. At the atomic scale, the properties of a material are determined by its electronic structure which requires proper treatment within quantum mechanics. Nowadays such an approach is often done within density functional theory which occurs in a large variety of approximations. They need to be explored and validated by even more sophisticated methods which may be limited by the computational effort for large system sizes. Such issues require efficient algorithms that can effectively be implemented on present day's computer hardware including parallelization. A realistic model for a complex material may require a relatively large system size whose treatment becomes computationally demanding. Keep in mind that for the comparison between theory and experiment, several idealizations must be assumed; for example, for structure (ideal $v s$. real), sample size (infinite $v s$. finite), stoichiometry (perfect $v s$. disordered), temperature ( $0 \mathrm{~K} v s$. finite temperature), property (ground state $v s$. excitations) all the way to quantum mechanics (approximated $v s$. valid). Another aspect is the time evolution of a system and its property (static vs. dynamics) leading to fields like molecular dynamics. In addition to ab-initio methods, coarse-grained molecular dynamics (MD) or Monte Carlo (MC) simulations may be the proper choice. In each case the question must be asked which aspects of the real system need to be taken into account and which can be ignored. In addition, often special results (for example spectra) must be calculated in order to allow a direct comparison with experimental data. Such details should be provided in good publications. The interdisciplinary nature of these issues is obvious, since the open challenges include chemistry, physics, mathematics, computer science and materials science. By a combined effort of experts from all these fields, a substantial process can be achieved: something we want to see. The characterization of a complex system by computational means can provide a basic understanding allowing predictions. Nowadays a systematic approach can replace the simple trial and error schemes. The systems of interest vary from molecules, crystals, surfaces to soft matter. With the computer power presently available, computations (or computer experiments) can explore a class of materials leading to new insight, provided a good model has been assumed and the essential approximations have been made.

\section{Computational Biology}

Following the classic principle that biology is nothing but the complex chemistry and physics of evolved systems, computational biology faces many of the same challenges as computational chemistry. Successful predictive modelling of biological systems requires appropriate simplification, to match the amount and detail of available experimental information and achieve algorithmic efficiency without losing predictive accuracy. In particular, biological phenomena occur over a wide range of spatial and temporal scales, from the subatomic to the ecosystem level, and from femtoseconds to the millennia required for evolutionary changes. A specific task of computational biology are the two main activities commonly referred to as bioinformatics, the study of the sequences of biological macromolecules, including entire genomes, and the analysis and management of huge postgenomic datasets, such as gene expression data (transcriptomics) and metabolite profiles 
(metabolomics). At the interface with computational chemistry, we find the still very active field of computational protein structure analysis, for example by molecular dynamics. On the other hand, recent advances in Synthetic Biology require novel computational approaches for the efficient engineering of new functional biosystems, including methods for biology-directed Computer-Aided Design and for the de novo construction of functional proteins, taking computational biology one step further towards computational engineering. All these aspects, and many other emerging topics of the rapidly developing science of computational biology, will be covered in this section.

\section{Computational Engineering}

Since the pioneering 18th century works of Euler and Lagrange, among others, who presented the governing equations for fluid flow and structural dynamics, it has been understood that theoretical analysis alone is incapable of providing solutions to many real engineering problems. Richardson's early 20th century work, on numerical weather prediction, presented the first steps into numerical approximation of partial differential equations, and interest in these methods has grown rapidly since, with progression accelerating in recent years due to the huge advances in cheap computing power available, to the point where numerical computation is standard in almost all areas of engineering and science. For example, finite element analysis forms the backbone of almost all structural design, both static and dynamic, and computational fluid dynamics is used extensively throughout fluid and aerodynamic design stages. Engineering applications present unique multidisciplinary requirements, so codes are often coupled, resulting in, for example, aeroelastic simulation tools. However, it is important to distinguish between numerical simulation tools and the prediction tools required in engineering. Brute force use of a coupled computational fluid dynamics/computational structural dynamics (CFD-CSD) code to find a flutter boundary, for example, is not efficient, and research into reduced order models or system identification methods for these systems is now common, leading to high fidelity computational methods being used in engineering design. Another research area of high activity is numerical optimisation, with many sophisticated deterministic, stochastic, heuristic, surrogate modelling, and gradient-based algorithms developed, with engineering applications often steering development in this field. All simulation tool developers must consider exploitation of the available computational hardware, and the development of parallel processing approaches has been led by computational engineers, particularly in the CFD area. New and emerging architectures offer huge potential performance benefits, and porting of computational engineering codes to graphics processing units (GPUs) has taken off in the last few years.

This section of the journal solicits works in all aspects of computational and numerical methods used in engineering-related research and design. In particular: Fundamental or novel numerical algorithms, including stability analysis of such; multi-physics algorithms; pre-, co-, and post-processing methods for numerical algorithms; novel meshing approaches; mesh free methods; system identification and reduced order models of engineering systems; optimisation methods; multidisciplinary optimisation; multi-fidelity modelling; uncertainty considerations; efficient parallel tools, and GPU exploitation. 


\section{Conclusion and Future Outlook}

The different computational methods covered by this journal provide a good basis to find better solutions in one field by looking at ideas that work well in other cases. Besides all the differences between chemistry, biology and engineering, these topics also have many challenges in common, when it comes to algorithms, parallel tools, numerical methods, accuracy, and efficiency. Therefore, practitioners in the various fields can learn from each other. In many applied papers the fundamental aspects of computational methods are hardly mentioned, but it is important that these are discussed for further improvements and innovations to be achieved. This open access journal provides the ideal forum for such an interdisciplinary dialog.

(C) 2013 by the authors; licensee MDPI, Basel, Switzerland. This article is an open access article distributed under the terms and conditions of the Creative Commons Attribution license (http://creativecommons.org/licenses/by/3.0/). 